\title{
Qualidade de vida da mulher com câncer de mama
}

\author{
Kátia Melissa Padilha, M.Sc.*; Carolina Pessoni**, Daniela Cervan Gomes**, Juliane Almeida da Silva**, \\ Rosangela Ferreira da Silva**, Rosangela Helena M. Barros**
}

*Professora da Universidade Paulista de Campinas, ${ }^{* *}$ Alunas do curso de graduação em Enfermagem da Universidade Paulista

\begin{abstract}
Resumo
Introdução: O câncer altera inúmeros aspectos no modo de vida do indivíduo. Objetivos: Realizar uma revisão sistemática de publicações científicas relacionadas a neoplasias mamárias, mastectomia e qualidade de vida; avaliar as relaçóes existentes entre as mesmas. Material e método: Pesquisa sobre a produção científica em periódicos indexados nos bancos de dados Lilacs e Medline, no período 2000-2005. Para a coleta dos dados foi utilizado um questionário dividido em: identificação do artigo; delineamento metodológico do estudo, qualidade de vida. A amostra foi de 42 artigos e 01 dissertação. Resultados: Apontam que a maior parte dos estudos apresentava deficiência de rigor metodológico na mensuraçáo da qualidade de vida e, além disso, não mencionou estratégias e condutas para melhoria da mesma. Conclusão: Os trabalhos permitem identificar e direcionar para a construção de novos projetos de pesquisa.
\end{abstract}

Palavras-chave: qualidade de vida, neoplasias mamárias, mastectomia.

\section{Abstract \\ Quality of life of woman with breast cancer}

Introduction: The cancer changes the way of the individual life in many aspects. Aims: To carry out a systematic literature review related to breast neoplasm, mastectomy and quality of life and to evaluate the relationship among each others. Methods: Study about the literature published in journals indexed in Lilacs and Medline databases from 2000 to 2005. It was used a questionnaire, divided as follows: identification of the article, design method and quality of life. The sample was comprised of 42 articles and 1 dissertation. Results: The results showed that most of the articles lacked consistency and methodological rigor in measuring quality of life among patients with mastectomy. Also, they did not mention strategies and behaviors for improving quality of life. Conclusion: The systematic review allows to identify and to give direction for new research projects.

Key-words: quality of life, breast neoplasm, mastectomy. 


\section{Resumen}

\section{Calidad de vida de la mujer con cáncer de mama}

Introducción: El cáncer altera el modo de vida del individuo. Objetivo: Realizar una revisión sistemática de publicaciones científicas acerca del tema neoplasias mamarias, mastectomía y calidad de vida y evaluar las relaciones existentes entre ellas. Objetivos: Realizar una revisión sistemática de publicaciones científicas existentes en periódicos indexados en los bancos de dados Lilacs y Medline en el período de 2000 al 2005. Para la recogida de datos fue utilizado un cuestionario, dividido en: identificación de artículos; delineamento metodológico del estudio; y calidad de vida. La muestra fue de 42 artículos y 01 disertación. Resultados: Apuntan que la mayor parte de los artículos presentaba inconsistencias y falta de rigor metodológico en la mensuración de la calidad de vida. Se verificó que la totalidad de los estudios no mencionaron estrategias y conductas para la mejoría en la calidad de vida de pacientes con mastectomía. Conclusión: Los estudios permiten identificar y orientar la elaboración de nuevos proyectos de investigación.

Palabras-clave: calidad de vida, neoplasias de la mama, mastectomía.

\section{Introdução}

O câncer, de modo geral, altera todos os aspectos da vida do indivíduo e pode levar a profundas alteraçóes no modo de viver. O sujeito, mesmo que estabilizado clinicamente, convive com a necessidade de acompanhamento regular e sob a contínua possibilidade de uma recidiva ou metástase [1].

$\mathrm{O}$ caráter mutilador do procedimento cirúrgico, aliado aos sintomas relacionados à quimioterapia, radioterapia e a hormonioterapia, favorecem o surgimento de muitas questóes na vida das pacientes, especialmente aquelas relacionadas à imagem corporal, e conseqüentemente, às alteraçóes em sua qualidade de vida [2-4].

$\mathrm{O}$ World Health Organization Quality of Life Group (WHOQOL Group) da Organização Mundial da Saúde define qualidade de vida como "a percepção do indivíduo de sua posição na vida no contexto da cultura e sistema de valores nos quais ele vive em relação aos seus objetivos, expectativas, padróes e preocupaçóes" [5,6].

Atualmente, devido à semelhança dos estudos desenvolvidos, alguns autores recomendam uma avaliação com critérios de qualidade e agrupados numa revisão sistemática e metanálise, o que garante inclusive uma maior confiabilidade e validade do resultado, garantindo uma informação de melhor qualidade [7-9].

Este estudo teve como objetivos realizar uma revisão sistemática de publicações científicas acerca do tema neoplasias mamárias, mastectomia e qualidade de vida; e avaliar as relaçóes existentes entre as mesmas.

\section{Material e métodos}

\section{Tipo de pesquisa}

Trata-se de uma pesquisa de revisão sistemática da literatura, reunindo os artigos em uma análise estatística, a metanálise. Por sintetizar estudos primários semelhantes, e de boa qualidade, é considerada o melhor nível de evidência para tomadas de decisões em questóes sobre intervençôes $[8,9]$.

\section{Coleta de dados}

A estratégia metodológica aplicada neste estudo propóe a utilização de alguns elementos básicos para uma revisão sistemática consistente: especificação do objeto, da estratégia de busca, definição dos critérios de inclusão e exclusão, aplicação de um questionário para avaliação qualitativa dos estudos [10].

\section{Levantamento bibliográfico}

A identificação de artigos se deu por meio de busca bibliográfica em bancos de dados da Literatura Latino-americana e do Caribe em Ciências da Saúde (Lilacs) e no National Library of Medicine (Medline). Os descritores utilizados foram: qualidade de vida, neoplasias mamárias e mastectomia; e seus equivalentes em inglês e espanhol: quality of life, mastectomy, breast cancer, calidad de vida, mastectomía e neoplasias mamarias.

\section{Critérios de inclusão/exclusão dos artigos}

Critérios de inclusão: artigos originais de pesquisa, e dissertaçóes e teses relacionadas, entre 2000 e 
2005; critérios de exclusão: artigos originais que envolvessem editoriais e relatos de caso, relativos à descrição de técnicas cirúrgicas, artigos de revisão bibliográfica.

\section{Questionário para avaliaçáo dos estudos}

O Questionário de Avaliação Qualitativa (QAQ) é composto por três itens: identificação do artigo, delineamento metodológico do estudo, qualidade de vida [10].

\section{Resultados e discussão}

Foram localizados 59 artigos. A leitura dos resumos resultou na seleçáo final de 42 artigos e 01 dissertação, totalizando 43 produções científicas.

Quanto ao ano de publicação dos artigos, observa-se que a maioria dos estudos foram publicados no ano de 2001 (Gráfico1).

Gráfico 1 - Distribuição dos resumos sobre qualidade de vida de pacientes mastectomizada, de acordo com ano de publicação. Campinas, 2006.

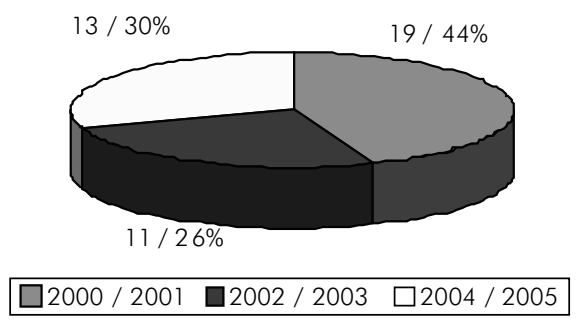

Em relação ao país onde foi realizado o estudo, os Estados Unidos da América (EUA) realizaram a maioria (10/43), seguido pelos países Europeus onde, juntos, foram responsáveis por $41,86 \%$ das publicaçôes. A produção científica nacional é responsável por apenas cinco dos 43 estudos pesquisados (Tabela I).

Tabela I - Distribuição dos estudos sobre QV de pacientes mastectomizada, de acordo com os países onde foram realizados os estudos. Campinas, 2006.

\begin{tabular}{lll}
\hline País do estudo & $\begin{array}{l}\mathrm{n}^{\circ} \text { de publi- } \\
\text { cações }\end{array}$ \\
\hline Países Europeus & 18 & 41,86 \\
EUA & 10 & 23,25 \\
Brasil & 05 & 11,63 \\
Oceania & 05 & 11,63 \\
Países da América Central & 03 & 6,98 \\
Outros países da América & 02 & 4,65 \\
do Sul & & 100 \\
\hline Total & 43 & \\
\hline
\end{tabular}

Um fator importante a ser considerado é que muitos instrumentos utilizados para mensurar qualidade de vida foram desenvolvidos nos Estados Unidos, sendo, portanto, aplicados mais facilmente em países de língua inglesa $[11,12]$.

Quando considerado as instituiçóes envolvidas no desenvolvimento dos estudos, $84 \%$ dos artigos foram promovidos por universidades, sendo todos realizados em hospitais. Apenas quatro estudos (9\%) foram realizados por instituiçóes hospitalares, e os restantes foram promovidos por instituiçóes de pesquisa (Gráfico 2).

Gráfico 2 - Distribuição dos resumos sobre qualidade de vida de pacientes mastectomizada, de acordo com as instituições envolvidas no desenvolvimento. Campinas, 2006.

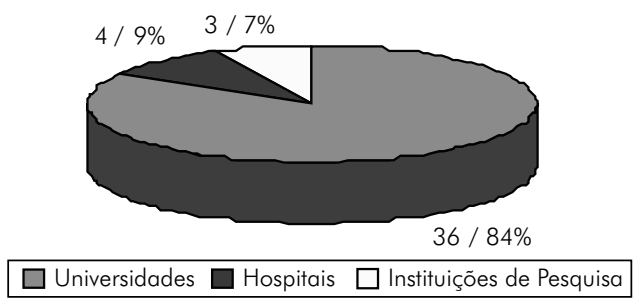

No que diz respeito à especialidade dos periódicos em que foram publicados os artigos selecionados, verificou-se que em $44,19 \%$ estavam relacionados à oncologia (Tabela II). Vale destacar que um dos trabalhos pesquisados trata-se de dissertação, portanto não foi incluído na categorização por especialidade, justificando o total de 42 periódicos distribuídos na Tabela II. No item "outros" foram agrupados as especialidades que foram citadas apenas duas ou uma vez, como, por exemplo, periódico de educação, ginecologia e radiologia.

Tabela II - Distribuição dos estudos sobre QV de pacientes mastectomizada, de acordo com a especialidade dos periódicos. Campinas, 2006.

\begin{tabular}{lll}
\hline Especialidade & $\begin{array}{l}\mathrm{n}^{\circ} \text { de publi- } \\
\text { cações }\end{array}$ & \\
\hline Oncologia & 19 & 45,24 \\
Cirurgia geral & 04 & 9,52 \\
Enfermagem & 04 & 9,52 \\
Qualidade de vida & 03 & 7,14 \\
Cirurgia plástica & 03 & 7,14 \\
Outros & 09 & 21,43 \\
\hline Total & 42 & 100 \\
\hline
\end{tabular}




\section{Características metodológicas do estudo}

No que se refere ao delineamento dos estudos, 23,26\% (10/43) tratava-se de estudo comparativo, $18,61 \%(08 / 43)$ realizaram estudos randomizados e com coorte prospectiva e em 9,31\% dos casos foram realizados estudos concomitantes (Tabela III).

Os estudos que investigaram a $\mathrm{QV}$ usando estudos comparativos relacionaram os métodos de procedimentos cirúrgicos e as terapias adjuvantes, ou seja, a quimioterapia e a radioterapia. A metodologia empregada nos estudos, com delineamento do tipo coorte prospectivo, vai ao encontro das recomendaçóes da literatura para estudos envolvendo qualidade de vida, pois em estudos de coorte prospectivo pode-se envolver com maior especificidade a questáo da qualidade de vida da população estudada [10]. Considerando a neoplasia mamária, há descrito na literatura que, na evolução da doença, há grande risco de metástase [13].

Tabela III - Distribuição dos estudos sobre qualidade de vida de pacientes mastectomizada, segundo o tipo de estudo realizado. Campinas, 2006.

\begin{tabular}{lll}
\hline Delineamento do estudo & $\begin{array}{l}N^{\circ} \text { de publica- } \\
\text { ções }\end{array}$ \\
\hline $\begin{array}{l}\text { Estudos observacionais } \\
\text { - Estudos de coorte pros- } \\
\text { pectivo }\end{array}$ & 08 & 18,61 \\
$\begin{array}{l}\text { - Estudos de coorte retros- } \\
\text { pectivo }\end{array}$ & 07 & 16,26 \\
- Estudos transversais & 06 & 13,95 \\
$\begin{array}{l}\text { Estudos experimentais } \\
\text { - Estudos randomizados }\end{array}$ & 08 & 18,61 \\
- Estudo comparativo & 10 & 23,26 \\
$\begin{array}{l}\text { Estudos observacionais }+ \\
\text { experimentais }\end{array}$ & \\
$\begin{array}{l}\text { Estudo de coorte + com- } \\
\text { parativo }\end{array}$ & 04 & 9,31 \\
\hline Total & 43 & 100 \\
\hline
\end{tabular}

Quanto ao método estatístico empregado, observou-se a utilização de mais de um método na maioria dos estudos. Metade dos estudos utilizou medidas não-paramétricas, como os coeficientes de correlação de Mann-Whitney, Kruskal-Wallis, Teste de Qui-Quadrado, Teste Exato de Fisher (55,81\% ou 24/43). Medidas paramétricas, como freqüência, medidas de posição e dispersão, foram aplicadas em 51,16\% (22/43) dos estudos, a Análise de Variância foi aplicada em três estudos. O alfa de Cronbach foi aplicado em apenas um estudo, referente à avaliação da confiabilidade.

Os métodos estatísticos empregados são os propostos na literatura $[8,14]$, no entanto, a literatura recomenda a avaliação da confiabilidade e da validade dos instrumentos na populaçáo estudada, mesmo que este instrumento já tenha sido validado em outras populaçóes $[8,15,16]$.

\section{Qualidade de Vida - QV}

No que se refere à utilização de instrumento para avaliaçáo de QV, 74,42\% (32/43) dos trabalhos utilizaram instrumentos para avaliação da QV na populaçáo estudada e 11 estudos avaliaram a QV de maneira subjetiva.

Dos 32 trabalhos analisados que utilizaram alguns instrumentos de QV, 27 (62,79\%) avaliaram a qualidade de vida aplicando instrumentos já validados, e cinco construíram e validaram um novo instrumento de medida (Gráfico 3).

Gráfico 3 - Distribuição dos tipos de intrumentos para mensuração da qualidade de vida aplicados nos estudos analisados. Campinas, 2006.

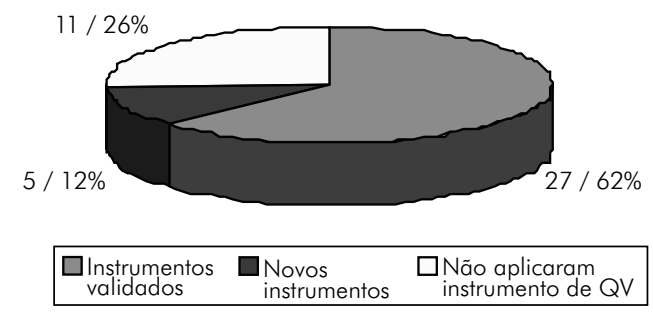

Tabela IV - Descrição dos instrumentos de qualidade de vida aplicados nos estudos analisados. Campinas, 2006.

\begin{tabular}{|c|c|}
\hline Instrumentos de Qualidade de Vida & $\mathrm{n}^{\circ}$ \\
\hline \multicolumn{2}{|l|}{ European Organization for Research and } \\
\hline $\begin{array}{l}\text { Treatment of Cancer - Quality of Life Ques- } \\
\text { tionnaire - EORTIC - QLQ C } 30\end{array}$ & 11 \\
\hline Medical Outcomes Study 36-item Short & 05 \\
\hline Form Health Survey - SF 36 & \\
\hline Functional Assessment of Cancer Therapy- & 05 \\
\hline Breast - FACT-B & \\
\hline $\begin{array}{l}\text { Short Form McGill Pain Questionnaire - } \\
\text { MPQ-SF }\end{array}$ & 02 \\
\hline Quality of Life Scale - QOLS & 01 \\
\hline $\begin{array}{l}\text { Breast Cancer-Specific Quality of Life Ques- } \\
\text { tionnaire - QLQ BR-23 }\end{array}$ & 01 \\
\hline Life Satisfaction Questionnaire - LSQ & 01 \\
\hline Quality of Life Index - IQV & 01 \\
\hline
\end{tabular}




\begin{tabular}{ll}
\hline Instrumentos de Qualidade de Vida & $\mathrm{n}^{\circ}$ \\
\hline Health-Related-Quality of Life - HRQL & 01 \\
Body Image After Breast Cancer Question- & 01 \\
naire - BIBCQ & \\
Breast Cancer Treatment Outcome Scale - & 01 \\
BCTOS & \\
RAND 36-item Health Survey - RAND-36 & 01 \\
\hline
\end{tabular}

A Tabela IV lista os 13 instrumentos de mensuração de QV utilizados nos estudos pesquisados. Todavia, alguns estudos utilizaram mais de um instrumento.

Ainda considerando os instrumentos de qualidade de vida, ou seja, 13 instrumentos, praticamente metade dos instrumentos aplicados $(46,15 \%$ ou 06/13) eram específicos para a população estudada, abordavam a qualidade de vida do paciente com câncer e 53,85\% (07/13) eram genéricos, avaliando a qualidade de vida geral (Gráfico 4).

Gráfico 4 - Distribuição dos 13 instrumentos QV. Campinas, 2006.

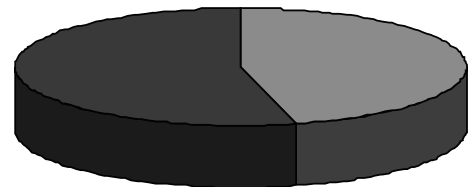

口Instrumento de QV Específico $\quad$ Instrumento de QV Genérico

O instrumento mais utilizado para mensuração da QV foi o European Organization for Research and Treatment of Cancer - Quality of Life Questionnaire - EORTIC - QLQ C 30 [17,18].

Quando avaliado a QV, notou-se maior escore de QV relacionado à dor, à imagem corporal e a fatores psicológicos, à diminuição da vitalidade, às alteraçóes no âmbito psicológico, à diminuição da capacidade física, à dificuldade nas relaçóes humanas, também, relacionado à sua autonomia, à espiritualidade, à sexualidade, à dificuldade de concentração.

\section{Conclusão}

Pode-se concluir que os trabalhos de revisão sistemática podem permitir identificar e direcionar a construção de novos projetos de pesquisa.

Este estudo evidenciou um crescimento importante da produção cientifica sobre o tema, nos últimos seis anos. No entanto, a maior parte dos estudos selecionados apresentava inconsistências e falta de rigor metodológico na mensuração da QV nessas pacientes. Além disso, verificou-se que quase a totalidade dos estudos não mencionou estratégias e condutas voltadas a melhoria da qualidade de vida. Espera-se, ainda, despertar o interesse dos profissionais da área da saúde, para incluir essa relevante temática nos projetos de pesquisa brasileiros, buscando, especialmente, as intervençóes com objetivo de melhor a qualidade de vida desta populaçáo.

\section{Referências}

1. Ferreira MLSM, Mamede MV. Representação do corpo na relaçáo consigo mesma após mastectomia. Rev Latinoam Enfermagem 2003;11(3):299-304.

2. Geiger AM, West CN, Nekhlyudov L, Herrinton LJ, Liu IL, Altschuler A et al. Contentment with quality of life among breast cancer survivors with and without contralateral prophylactic mastectomy. J Clin Oncol 2006;24(9):1350-6.

3. Ishiyama H, Niino K, Hosoya T, Hayakawa K. Results of a questionnaire survey for symptom of late complications caused by radiotherapy in breast conserving therapy. Breast Cancer 2006;13(2):197-201.

4. Michelone APC, Santos VLCG. Qualidade de vida de adultos com câncer colorretal com e sem ostomia. Rev Latinoam Enfermagem 2004;12(6):875-83.

5. Whoqol Group. The World Health Organization Quality of Life Assessment (WHOQOL): position paper from the World Health Organization. Special Issue "Quality of Life". Soc Sci Med 1995;10(14):3-9.

6. Cella DF, Tulsky DS. Measuring the quality of life today: methodological aspects. Oncology 1990;4(5):2938 .

7. Bork AMT. Enfermagem baseada em evidências. Rio de Janeiro: Guanabara Koogan; 2005. 365p.

8. Hulley SB, Cummings SR, Browner WS, Grady D, Hearst N, Newman TB. Delineando a Pesquisa Clínica: uma abordagem epidemiológica. 2a.ed. Porto Alegre: Artmed; 2003. 374p.

9. Atallah AN, Castro AA. Evidências para melhores decisóes clínicas. São Paulo: Lemos; 1998.

10. Moreno AB, Lopes CS. Avaliaçáo da qualidade de vida em pacientes laringectomizados: uma revisão sistemática. Cad Saúde Pública 2002;18(1):81-92.

11. Canini SRMS, Reis RB, Pereira LA, GirE, Pelá NTR. Qualidade de vida de indivíduos com HIV/AIDS: uma revisão de literatura. Rev Latinoam Enfermagem 2004;12(6):940-5.

12. Kimura M. Tradução para o português e validação do "Quality of Life Index" de Ferrans e Powers [tese]. São Paulo: Universidade de São Paulo; 1999.

13. Inca - Instituto Nacional do Câncer, 2005. [citado 2006 Abr 09]. Disponível em URL: http://www. inca.gov.br.

14. Franco LJ, Passos ADC. Fundamentos de epidemiologia. Barueri: Manole; 2005.

15. Pasquali L. Instrumentos Psicológicos: manual prático de elaboração. Brasília: LabPAM; 1999. 306p

16. Strainer DL, Norman GR. Health measurement scales: a practical guide to their development and use. 2nd ed. New York: Oxford University Express; 2001.

17. Minayo MCS, Hartz ZMA, Buss PM. Qualidade de vida e saúde: um debate necessário. Ciên Saúde Coletiva 2000;5(1):7-18.

18. Conde DM, Neto AMP, Júnior RF, Aldrigh JM. Qualidade de vida de mulheres com câncer de mama. Rev Bras Ginecol Obstet 2006;28(3):195-204. 